\title{
ON HOLOMORPHIC FAMILIES OF RATIONAL MAPS: FINITENESS, RIGIDITY AND STABILITY
}

\author{
Hiroshige Shiga
}

\begin{abstract}
We consider holomorphic families of rational maps from the viewpoint of complex dynamics.

First, we consider some classes of families of rational maps which satisfy a certain stability condition. We show a finiteness theorem for such holomorphic families of rational maps parameterized by a Riemann surface of finite type.

Next, we consider the monodromy of quasiconformally stable holomorphic families of rational maps over a punctured disk, and study the action of the monodromy on the Julia set.
\end{abstract}

\section{Introduction}

D. Sullivan proposed a project to look up similar phenomena between the theory of Kleinian groups and the theory of complex dynamics of rational maps (cf. $[\mathrm{Su}]$ ). The list of collected phenomena is called "Sullivan's dictionary". For example, the number of generators of finitely generated Kleinian groups corresponds to the degree of rational maps, and as many computer graphics show, the Julia sets of rational maps look like the limit sets of Kleinian groups. Furthermore, Teichmüller spaces for rational maps are defined and it is shown that they have the similar properties to Teichmüller spaces of Kleinian groups.

In this paper, we consider holomorphic families of rational functions parameterized by Riemann surfaces. From the standpoint of Sullivan's dictionary, we find a natural correspondence of families of Riemann surfaces to those of rational maps. Actually, if we consider a holomorphic family of (hyperbolic) Riemann surfaces, then it produces a holomorphic assignment from the parameter space to quasi-Fuchsian groups uniformizing the Riemann surfaces. According to the dictionary, quasi-Fuchsian groups correspond to rational maps. Therefore, we consider that holomorphic families of rational maps correspond to those of Riemann surfaces.

First of all, we recall the following finiteness theorem for holomorphic families of Riemann surfaces parameterized by a Riemann surface of finite type.

Received March 7, 2000; revised November 14, 2000. 
THEOREM 1.1 (Parshin-Arakelov). Let $X$ be a Riemann surface of finite type. Then, there are only finitely many non-isomorphic and locally non-trivial holomorphic families of Riemann surfaces of fixed finite type $(g, n)$ with $2 g-2+n>0$ over $X$.

This theorem was first conjectured by Shafarevich and it is called Shafarevich conjecture in the function field case. Parshin proved it for a compact Riemann surface $X$ and holomorphic families of Riemann surfaces of type $(g, 0)(g \geq 2)$. Arakelov proved it for $X$ of finite type and holomorphic families of Riemann surfaces of type $(g, 0)(g \geq 2)$. In [ISh], the theorem with the condition as above is shown by using the theory of Teichmüller spaces and Kleinian groups.

Once the finiteness theorem is established, uniform boundedness of the number of families is an interesting question, that is, if the Riemann surface $X$ varies in the moduli space, then whether or not there exists an upper bound of the numbers of holomorphic families over $X$ which does not depend on $X$.

Recently, we have obtained a partial answer to this problem ([Sh2]).

THEOREM 1.2. Let $X$ be a Riemann surface of type $(p, k)$. Then, the number of non-isomorphic and locally non-trivial holomorphic families of Riemann surfaces of type $(g, n)$ over $X$ is uniformly bounded if $(g, n)=(0, n),(1,1),(1,2)$ or $(2,0)$ $(n \geq 4)$.

As a corresponding problem in holomorphic families of rational functions, we shall consider the following one.

Problem. Let $X$ be a Riemann surface of type $(g, n)$. Then, are there at most finitely many non-isomorphic and locally non-trivial holomorphic families of rational maps of degree $d \geq 2$ ? And if it is finite, then is there an upper bound of the number of families which depends only on $d, g$ and $n$ ?

Unfortunately, this problem has a negative answer. Namely, we can find a Riemann surface of finite type over which there are infinitely many nonisomorphic and locally non-trivial holomorphic families of rational maps. However, we shall show that if we restrict our families to ones which satisfy a certain reasonable condition, then the number of families over a Riemann surface of finite type is finite.

Secondly, we consider the monodromy of holomorphic families.

In case of holomorphic families of Riemann surfaces, all Riemann surfaces which appear in fibers of the family are quasiconformally equivalent to each other. So, analytic continuations of closed curves in the parameter space determines a (homotopy class of) quasiconformal self mapping of the Riemann surfaces. It induces a homomorphisms of the fundamental group of the surface to the mapping class group which is called monodromy. It is known that the monodromy groups play an important role in holomorphic families of Riemann surfaces (cf. [ISh], [Sh2]).

On the other hand, in case of holomorphic families of rational maps with 
the same degree, all rational maps in a family are not necessarily quasiconformally equivalent to each other. In this paper, we consider holomorphic families of rational maps over the punctured disk $\Delta^{*}=\{0<|z|<1\}$, and assume that they are obtained by quasiconformal deformations. We shall show that under a condition the monodromy for a simple closed curve in $\Delta^{*}$ around the origin is of infinite order (Theorem 4.7). It is a generalization of a result in a paper of McMullen [Mc2]. The result corresponds to the fact that for a holomorphic family of Riemann surfaces over the punctured disk, the monodromy for a simple closed curve around the origin is a Dehn twist if the family does not have an analytic continuation to the origin $([\mathrm{I}])$. Our proof deeply depends on the theory of Teichmüller spaces of complex dynamics which is developed in a paper of McMullen and Sullivan [MSu].

We give some examples of families for Theorem 4.7 in the last section.

As for terminologies (e.g. Julia set, Fatou set etc.) and fundamental facts of complex dynamics of rational maps, we refer to $[\mathrm{B}]$ and $[\mathrm{CG}]$.

The author thanks Curt McMullen for his helpful comments and suggestions. He also thanks the referee who carefully read the manisucript and gave useful comments.

\section{Holomorphic families of rational maps}

Throughout this paper, the degree $d$ of a rational map is not less than 2 . We denote by $R_{a t}$ the space of rational functions of degree $d$. Since $R a t_{d}$ is identified with $\boldsymbol{P}^{2 d+2}-\{$ some algebraic varieties $\}$, it has a natural complex structure.

Definition 2.1. Let $\left\{R_{\lambda}\right\}_{\lambda \in M}$ be a family of rational functions parameterized by a complex manifold $M$. Then it is called a holomorphic family over $M$ if the assignment

$$
M \ni \lambda \mapsto R_{\lambda} \in R_{a t}
$$

is holomorphic.

Definition 2.2. Let $\left\{R_{\lambda}^{(j)}\right\}_{\lambda \in M}(j=1,2)$ be holomorphic families of rational maps over a complex manifold $M$. They are called isomorphic if there exists a holomorphic mapping $\phi$ from $M$ to $\operatorname{PSL}(2, C)$ such that

$$
\phi(\lambda) \circ R_{\lambda}^{(1)}=R_{\lambda}^{(2)} \circ \phi(\lambda) \text { for all } \lambda \in M .
$$

A holomorphic family $\left\{R_{\lambda}\right\}_{\lambda \in M}$ of rational maps over $M$ is called locally trivial if for each point $z \in M$ there exist a neighbourhood $U$ of $z$ and a rational map $R_{0}$ such that the restricted family $\left\{R_{\lambda}\right\}_{\lambda \in U}$ on $U$ is isomorphic to a trivial family $\left\{R_{0}\right\} \times U$ over $U$.

First, we construct a Riemann surface $X$ of finite type which gives a negative answer to the problem in the introduction. Namely, $X$ admits infinitely many non-isomorphic and locally non-trivial holomorphic families of rational maps. 
Example 2.1. Let $\bar{X}$ be a compact Riemann surface and $R$ be an arbitrary rational map. For each non-constant meromorphic function $f(\lambda)$ on $\bar{X}$, consider an assignment

$$
X=\bar{X}-Z \ni \lambda \mapsto R_{\lambda}=f(\lambda) R \in R_{a t},
$$

where $Z$ be the set of zeros and poles of $f$ on $\bar{X}$. Then above assignment determines a holomorphic family over $X$. Obviously, $X$ is a Riemann surface of finite type.

We can find a rational map $R$ so that the above family is locally non-trivial. For example, if $R$ has at least three critical points, then the family $\left\{R_{\lambda}\right\}_{\lambda \in X}$ is not locally trivial. Indeed, if it is locally trivial, then there exists a $\gamma \in \operatorname{PSL}(2, C)$ such that

$$
\gamma \circ R_{\lambda}(z)=R_{\lambda^{\prime}} \circ \gamma(z)
$$

for $\lambda^{\prime}(\neq \lambda)$ in a small neighbourhood of $\lambda$ in $X$. Since $\gamma$ maps the set of critical points of $R_{\lambda}$ to that of $R_{\lambda^{\prime}}$ from the above relation and all of critical points are constants with respect to $\lambda$ from (2.1), $\gamma$ fixes these critical points. Hence we see that $\gamma$ must be the identity because the set of critical points contains three points. On the other hand, $R_{\lambda^{\prime}}$ is actually different from $R_{\lambda}$ because $f$ is non-constant. Thus we have a contradiction and we conclude that the family is not locally trivial. $\left\{R_{\lambda}^{(n)}\right\}_{\lambda \in X}$ by

Similarly, we have infinitely many locally non-trivial holomorphic families

$$
X \ni \lambda \mapsto R_{\lambda}^{(n)}=f(\lambda)^{n} R \in \operatorname{Rat}_{d} \quad(n=2,3, \ldots) .
$$

By the similar reason as above, we verify that a family $\left\{R_{\lambda}^{(n)}\right\}_{\lambda \in X}$ is not isomorphic to $\left\{R_{\lambda}^{(m)}\right\}_{\lambda \in X}$ if $n \neq m$. Therefore the Riemann surface $X$ admits infinitely many locally non-trivial and non-isomorphic holomorphic families.

Here, we reconsider the problem for some classes of holomorphic families of rational maps which appear in many situations. In fact, there are several important classes of holomorphic families (cf. [MSu]). We recall the definition of stable families and quasiconformally stable families.

Definition 2.3 (Stability). Let $\left\{R_{\lambda}\right\}_{\lambda \in M}$ be a holomorphic family of rational maps over a complex manifold $M$. Then it is stable (resp. quasiconformally stable) if for each point $\lambda \in M$ there exists a neighbourhood $U$ of $\lambda$ such that the action of $R_{\lambda}$ on its Julia set $J\left(R_{\lambda}\right)$ (resp. on $\hat{C}$ ) is quasiconformally conjugate to the action of $R_{\lambda^{\prime}}$ on the Julia set $J\left(R_{\lambda^{\prime}}\right)$ (resp. on $\hat{C}$ ) for each $\lambda^{\prime} \in$ $U$. More precisely, there exists a quasiconformal self-mapping $f_{\lambda^{\prime}}$ of $\hat{\boldsymbol{C}}$ for each $\lambda^{\prime} \in U$ such that $f_{\lambda^{\prime}}$ depends holomorphically on $\lambda^{\prime}$ and

$$
f_{\lambda^{\prime}} \circ R_{\lambda}(z)=R_{\lambda^{\prime}} \circ f_{\lambda^{\prime}}(z)
$$

holds for every $z \in J\left(R_{\lambda}\right)$ (resp. $z \in \hat{\boldsymbol{C}}$ ).

Stable families are well investigated by many authors. It is known that there are some equivalent conditions for stable families. 
Proposition 2.1. Let $\left\{R_{\lambda}\right\}_{\lambda \in M}$ be a holomorphic family of rational maps over a complex manifold $M$. Let $\lambda_{0}$ be an any point in $M$. Then the following conditions are equivalent.

1. $\left\{R_{\lambda}\right\}_{\lambda \in M}$ is a stable family.

2. The number of attracting cycles of $R_{\lambda}$ is locally bounded at $\lambda_{0}$.

3. The Julia set $J_{\lambda}$ of $R_{\lambda}$ depends continuously on $\lambda$ (in the Hausdorff topology) on a neighbourhood of $\lambda_{0}$.

McMullen considers stable holomorphic families of rational maps over a "nearly" compact complex manifold.

Proposition 2.2 (McMullen [Mc2]). Let $M$ be a complex manifold on which there do not exist non-constant bounded holomorphic functions. Then, any stable family of rational maps over $M$ is either affine or trivial.

In the above proposition, a family of rational maps is called affine if the family consists of affine rational maps. Affine rational maps are rational maps constructed by the following way.

Take a torus $T=\boldsymbol{C} / \Lambda$ determined by an integral lattice $\Lambda$ and consider a multiplicative map $g_{\alpha}: z \mapsto \alpha z$ for some $\alpha \in C$ with $|\alpha|>1$. We assume that $g_{\alpha}$ is equivariant with respect to the action of $\Lambda$. (Take $\alpha$ in $\boldsymbol{Z}$, for example.) Since $g_{\alpha}$ is also equivariant to the involution $E: z \mapsto-z$, it is projected to a holomorphic mapping of $\boldsymbol{C} /\{\Lambda, E\}=\hat{\boldsymbol{C}}$ onto itself. Therefore, the projected mapping is a rational map. We call rational functions obtained by this way affine rational maps.

Note that the degree of an affine rational map obtained from $g_{\alpha}$ as above is $|\alpha|^{2}$ and that the Julia set of any affine rational map is $\hat{\boldsymbol{C}}$.

From Proposition 2.2, we have a finiteness theorem for stable holomorphic families of rational maps over a Riemann surface of finite type.

COROLlaRY 2.3. There are only finitely many non-isomorphic and locally non-trivial holomorphic stable families of rational maps of degree d over a Riemann surface $X$ of type $(g, n)$. Moreover, there exists a number $N=N(g, n, d)$ depending only on $g, n$ and $d$ such that the number of non-isomorphic and locally nontrivial stable families over $X$ is less than $N$.

Proof. Since $X$ admits no non-constant bounded holomorphic functions, any locally non-trivial holomorphic family $\left\{R_{\lambda}\right\}_{\lambda \in X}$ of rational maps over $X$ must be affine from Proposition 2.2.

Let $\alpha_{\lambda}$ and $T_{\lambda}$ be a constant and a torus which define an affine rational map $R_{\lambda}$ for $\lambda \in X$. Let $\pi_{\lambda}: T_{\lambda} \rightarrow \hat{\boldsymbol{C}}$ be the ramified covering map induced by $E$ above. Then, the postcritical set $P\left(R_{\lambda}\right)$ defined by

$$
P\left(R_{\lambda}\right)=\overline{\bigcup_{c \in C\left(R_{\lambda}\right), n>0} R_{\lambda}^{n}(c)}
$$


coincides with the set of the critical values of $\pi_{\lambda}$, where $C\left(R_{\lambda}\right)$ is the set of critical points of $R_{\lambda}$. Hence, $P\left(R_{\lambda}\right)$ consists of four points and any two points of them do not collide for any $\lambda \in X$. Since $R_{\lambda}$ depends holomorphically on $\lambda$, so does each point of $P\left(R_{\lambda}\right)$. By taking a finite covering of $X$ if necessary, we may assume that these four points are holomorphic mapping of $X$ to the sphere $\hat{\boldsymbol{C}}$ and three of them are 0,1 and $\infty$ by taking a conjugation via a Möbius transformation. Thus, the rest of them, say $c(\lambda)$, defines a holomorphic mapping of $X$ to $C-\{0,1\}$, and it determines $\pi_{\lambda}$ and $T_{\lambda}$. It is known that there are only finitely many holomorphic mappings of $X$ to $\boldsymbol{C}-\{0,1\}$ (see Proposition 3.4). Thus, we verify that there are only finitely many possibilities for $c(\lambda)$ and for $T_{\lambda}$ if $c(\lambda)$ is non-constant with respect to $\lambda$.

As for $\alpha_{\lambda}$, it is easily seen that there are only finitely many possibilities for $\alpha_{\lambda}$ because $d=\left|\alpha_{\lambda}\right|^{2}$. Therefore, we verify that the number of non-isomorphic and locally non-trivial stable holomorphic families of rational maps of degree $d$ over $X$ is bounded by some number depending only on $g, n$ and $d$.

\section{Weakly stable families and finiteness theorem}

We have shown a finiteness theorem of stable holomorphic families of rational maps over a Riemann surface $X$ of finite type. But, as we have seen, stable families over a Riemann surface of finite type are strongly restrictive, i.e., trivial or affine. In this section, we give a finiteness theorem for holomorphic families of rational maps belonging to a certain class which is a generalization of stable families.

DeFinition 3.1 (Weakly stable family). Let $\left\{R_{\lambda}\right\}_{\lambda \in M}$ be a holomorphic family of rational maps of degree $d$ over a complex manifold $M$ and $k$ a positive integer. Then, it is called a weakly $k$-stable family over $M$ if it satisfies the following condition.

- There exists some period $p$ such that the set of periodic points $E_{\lambda}$ of $R_{\lambda}$ with period $p$ consists of exactly $k$ points for every $\lambda \in M$ (cf. [CG] III Lemma 2.5).

If a holomorphic family $\left\{R_{\lambda}\right\}_{\lambda \in M}$ is weakly $k$-stable, then we see that there exists a neighbourhood $U$ of $\lambda \in M$ such that each points of $E_{\lambda^{\prime}}$ is holomorphic with respect to $\lambda^{\prime} \in U$.

We can show that any stable family is weakly stable.

Proposition 3.1. Let $\left\{R_{\lambda}\right\}_{\lambda \in M}$ be a stable holomorphic families of rational maps of degree d over a complex manifold $M$. Then, it is a weakly $k$-stable family over $M$ for a sufficiently large order $k$.

Proof. From a characterization of stable families (Proposition 2.1), we see that periods of attractive cycles of the family are uniformly bounded. Therefore, any indifferent periodic point is stable because if not, the multiplier moves holomorphically and it produces a new attractive periodic point. Thus, if we 
take periodic cycles for a sufficiently large period $p$, all of then are repelling and they are in the Julia sets. Since a stable family has a quasiconformal conjugacy on the Julia sets and the quasiconformal mappings depend holomorphically on $\lambda \in M$, we verify that the family is weakly stable.

For any holomorphic family $\left\{R_{\lambda}\right\}_{\lambda \in M}$ of rational maps over a complex manifold $M$, we denote by $M^{\text {stable }}$ the set of stable parameters $\lambda$ in $M$ and by $M_{k}^{\mathrm{w}-\text { stable }}$ the set of weakly stable parameters $\lambda$ with order $k$ in $M$. From Proposition 3.1 $M^{\text {stable }} \subset \bigcup_{k} M_{k}^{\mathrm{w}-\text { stable }}$. It is known that $M^{\text {stable }}$ is an open dense

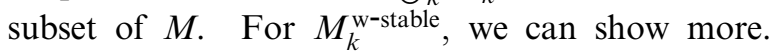

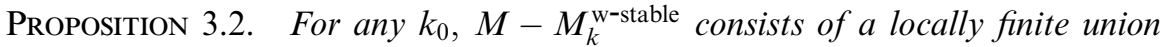
of analytic varieties on $M$ for some $k \geq k_{0}$.

Proof. Let us consider an algebraic equation

$$
R_{\lambda}^{p}(z)-z=0
$$

for some $p \in N$. On a neighbourhood $U$ of $\lambda_{0} \in M$, we denote by $N_{U}$ the maximal number of the solutions of (3.1) in $U$. Since any collision of solutions of (3.1) gives algebraic equations of coefficients of (3.1), the complement of the set of $\lambda \in U$ for which the number of solutions of (3.1) is $N_{U}$ is a finite union of analytic varieties on $U$. If we take $p$ sufficiently large, then we have $k=N_{U} \geq$ $k_{0}$. Hence the set of $\lambda \in U$ for which the number of the solutions of (3.1) is $k$ gives a weakly $k$-stable family. Thus, we complete the proof.

There is a weakly stable family of rational maps which is not stable. Thus, the weakly stability is actually a generalization of the stability.

Example 3.1 (A weakly stable family which is not stable.). Let $\bar{X}$ be a compact Riemann surface and $f$ be a non-constant meromorphic function on $\bar{X}$. We take a rational function $R(z)$ of degree $d$ and consider a family defined by

$$
\lambda \mapsto f(\lambda) R(z)=R_{\lambda}(z)
$$

over $\bar{X}$ as Example 2.1. We assume that the Julia set $J\left(R_{\lambda}\right)$ of $R_{\lambda}(z)=f(\lambda) R(z)$ is not the sphere $\hat{C}$ for all $\lambda$ in some open set in $\bar{X}$. (Take a polynomial, for example.) Let $Z$ be the zeros and the poles of $f$ in $\bar{X}$. We may also assume that the family $\left\{R_{\lambda}\right\}_{\lambda \in \bar{X}-Z}$ is not locally trivial as Example 2.1.

Now, we consider the periodic points of $R_{\lambda}$ with some period $p$. They are solutions of an algebraic equation

$$
R_{\lambda}^{p}(z)-z=0 .
$$

Therefore, the number of the periodic points is constant except at most finitely many points $Y$ on $\bar{X}$, because a point in $Y$, which makes a collision of these periodic points, is a zero of some meromorphic functions on $\bar{X}$. Hence, $X=$ 
$\bar{X}-Y-Z$ is a Riemann surface of finite type and the family $\left\{R_{\lambda}\right\}_{\lambda \in X}$ is a weakly stable family from the construction. But it is not stable. Indeed, if the family is stable, then it is an affine family from Proposition 2.2, because it is not trivial. Thus, the Julia set $J\left(R_{\lambda}\right)$ for any rational map $R_{\lambda}$ for $\lambda \in X$ is the sphere $\hat{C}$. It contradicts our choice of $R$. Hence this is our desired example.

We extend Corollary 2.3 to weakly stable families.

THEOREM 3.3. Let $X$ be a Riemann surface of type $(g, n)$. We denote by $N(X, d, k)$ the number of non-isomorphic and locally non-trivial weakly $k$-stable holomorphic families of rational maps of degree $d$ over $X$. If $k \geq 3\left(2 d^{2}+1\right)$, then there exists an $N=N(g, n, d, k)$ depending only on $g, n, d$ and $k$ such that $N(X, d, k) \leq N$ for all $X$ of type $(g, n)$.

Proof. Let $\left\{R_{\lambda}\right\}_{\lambda \in X}$ be a locally non-trivial weakly $k$-stable holomorphic family of rational maps over $X$ for $k \geq 3\left(2 d^{2}+1\right)$ and denote by $E_{\lambda}=\left\{a_{1}(\lambda)\right.$, $\left.a_{2}(\lambda), \ldots, a_{k}(\lambda)\right\}$ the finite set associated to $R_{\lambda}$ in the definition of weakly stable family. From the definition, each $a_{j}(\lambda)$ is holomorphic on an open set $U$ in $X$. Hence, taking a smooth covering $\tilde{X}$ over $X$, we verify that each $a_{j}(\lambda)$ is lifted to a holomorphic function on $\tilde{X}$.

Indeed, take a base point $\lambda_{0} \in U$ and consider an analytic continuation of $a_{j}(\lambda)$ along a $c \in \pi_{1}\left(X, \lambda_{0}\right)$. We denote by $a_{j}^{c}(\lambda)$ a holomorphic germ at $\lambda_{0}$ obtained by the analytic continuation of $a_{j}(\lambda)$ along $c$. Since $R_{\lambda}\left(E_{\lambda}\right)=E_{\lambda}$, we verify that $a_{j}^{c}(\lambda) \in E_{\lambda}$. Therefore, we have a homomorphism $\rho: \pi_{1}\left(X, \lambda_{0}\right) \rightarrow \mathscr{S}_{k}$ by

$$
a_{\rho(c)(j)}(\lambda)=a_{j}^{c}(\lambda)
$$

for $c \in \pi_{1}\left(X, \lambda_{0}\right)$, where $\mathscr{S}_{k}$ is the $k$-symmetric group. The kernel Ker $\rho \subset$ $\pi_{1}\left(X, \lambda_{0}\right)$ determines a finite-sheeted smooth covering $\tilde{X}$ over $X$, and the index $\left[\pi_{1}\left(X, \lambda_{0}\right): \operatorname{Ker} \rho\right]$ is the number of the sheets, which is not greater than $k !$. From the construction, each $a_{j}(\lambda)$ is lifted to an analytic function $A_{j}(\lambda)$ on $\tilde{X}$. Note that the genus $\tilde{g}$ and the number of punctures, $\tilde{n}$ of $\tilde{X}$ have an upper bound depending only on $g, n$ and $k$.

Taking conjugations via Möbius transformations, we may assume that $A_{1}(\lambda)=0, A_{2}(\lambda)=1$ and $A_{3}(\lambda)=\infty$ for all $\lambda \in \tilde{X}$. Therefore, $A_{4}(\lambda), \ldots, A_{k}(\lambda)$ are holomorphic mappings from $\tilde{X}$ to $C-\{0,1\}$. Here, we note the following fact (cf. [Mc2], [Sh2]).

Proposition 3.4. Let $X$ be a Riemann surface of type $(g, n)$. Then, there are only finitely many holomorphic functions on $X$ which do not take 0 and 1. Moreover, the number of these holomorphic functions on $X$ has an upper bound $M(g, n)$ which depends only on $g$ and $n$.

Hence, if some $A_{j}(\lambda)$ is not a constant on $\tilde{X}$, there are only finitely many possibilities of $A_{j}(\lambda)$. Let $\operatorname{Con}(k)$ be the set of $j \in\{1,2, \ldots, k\}$ so that $A_{j}(\lambda)$ is a constant function on $\tilde{X}$ and $N \operatorname{Con}(k)=\{1,2, \ldots, k\}-\operatorname{Con}(k)$. 
Lemma 3.5. Let $R_{j}(j=1,2)$ be rational maps of degree $d$. If there are $(2 d+1)$ points $z_{1}, z_{2}, \ldots, z_{2 d+1}$ in $\hat{\boldsymbol{C}}$ such that $R_{1}\left(z_{i}\right)=R_{2}\left(z_{i}\right)(i=1,2, \ldots$, $2 d+1)$, then $R_{1}=R_{2}$.

Proof of the lemma. By taking a conjugation via a Möbius transformation, we may assume that $z_{j}, R_{1}\left(z_{j}\right), R_{2}\left(z_{j}\right)$ are in $C(j=1,2, \ldots, 2 d+1)$. Since the degree of a rational map $R=R_{1}-R_{2}$ is less than $(2 d+1), R$ is at most $2 d$ valent holomorphic mapping of $\hat{\boldsymbol{C}}$ into itself. Hence $R$ must be identically zero because it has $(2 d+1)$ zeros.

Since $R_{\lambda}\left(E_{\lambda}\right)=E_{\lambda}, R_{\lambda}$ determines an element $\sigma \in \mathscr{S}_{k}$ with

$$
R_{\lambda}\left(A_{j}(\lambda)\right)=A_{\sigma(j)}(\lambda) \quad(j=1,2, \ldots, k) .
$$

Then, for $j \in\{1,2, \ldots, k\}$ the following cases are possible.

P1: Both $j$ and $\sigma(j)$ belong to $\operatorname{Con}(k)$.

P2: Both $j$ and $\sigma(j)$ belong to $N C o n(k)$.

P3: $j$ belongs to $N C o n(k)$ but $\sigma(j)$ belongs to $\operatorname{Con}(k)$.

P4: $j$ belongs to $\operatorname{Con}(k)$ but $\sigma(j)$ belongs to $N \operatorname{Con}(k)$.

1. If there are $(2 d+1)$ numbers $j \in\{1,2, \ldots, k\}$ which satisfy $\mathbf{P 1}$, then $R_{\lambda}$ is locally constant with respect to $\lambda$ from Lemma 3.5. This means $\left\{R_{\lambda}\right\}_{\lambda \in \tilde{X}}$ is locally trivial. Hence, we do not need to consider such a case.

2. Assume that there are $(2 d+1)$ numbers $j \in\{1,2, \ldots, k\}$ which satisfy P2. From Proposition 3.4, there are only finitely many possibilities for $A_{j}(\lambda)$ and $A_{\sigma(j)}(\lambda)=R_{\lambda}\left(A_{j}(\lambda)\right)$. Hence, we verify that there are also finitely many possibilities for $R_{\lambda}$ from Lemma 3.5, and it shows the finiteness of families.

3. Suppose that $j$ satisfies P3. From Proposition 3.4, there are only finitely many possibilities for $A_{j}(\lambda)$. But there may be infinitely many possibilities for a constant function $A_{\sigma(j)}(\lambda)$. We consider $\sigma^{2}(j)$.

(a) If $\sigma^{2}(j)$ belongs to $\operatorname{Con}(k)$, then the relationship between $\sigma(j)$ and $\sigma^{2}(j)$ is P1. Thus, we can apply the first argument above and we conclude that the family is locally trivial if there are $(2 d+1)$ such numbers $j$.

(b) If $\sigma^{2}(j)$ belongs to $N C o n(k)$, then the relationship of $j$ and $\sigma^{2}(j)$ is P2. There are only finitely many possibilities for $A_{j}(\lambda)$ and $A_{\sigma^{2}(j)}(\lambda)$ $=R_{\lambda}^{2}\left(A_{j}(\lambda)\right)$. Thus, we can apply the second argument above for $R_{\lambda}^{2}$. Since $R_{\lambda}^{2}$ is a rational map of degree $d^{2}$, if there are $\left(2 d^{2}+1\right)$ such numbers $j$, we verify the finiteness of $R_{\lambda}^{2}$. And it also shows the finiteness of $R_{\lambda}$. $R_{\lambda}$.

4. If $j$ satisfies $\mathbf{P 4}$, by the similar argument as (3), we verify the finiteness of

From our assumption, $k \geq 3\left(2 d^{2}+1\right)$, we see that one of these cases occurs in $E_{\lambda}$ and the finiteness is shown as above.

Moreover, if we find $\left(2 d^{2}+1\right)$ numbers $j$ such that $j, \sigma^{2}(j) \in N \operatorname{Con}(k)$ or $(2 d+1)$ numbers $j$ such that $j, \sigma(j) \in N \operatorname{Con}(k)$, then we conclude that the number 
of such families is at most $M(\tilde{g}, \tilde{n})^{2 d^{2}+1}$ or $M(\tilde{g}, \tilde{n})^{2 d+1}$, where $M(\tilde{g}, \tilde{n})$ is a constant given in Proposition 3.4 for $\tilde{X}$. Hence, we complete the proof of Theorem 3.3.

\section{Monodromy of holomorphic families of rational maps}

We review the theory of Teichmüller spaces of rational maps developed in $[\mathrm{MSu}]$. See $[\mathrm{MSu}]$ for the details.

Let $R$ be a rational mapping of degree $d \geq 2$. We consider quasiconformal maps $\varphi: \hat{\boldsymbol{C}} \rightarrow \hat{\boldsymbol{C}}$ such that $R^{\varphi}=\varphi \circ R \circ \varphi^{-1}$ are rational maps. Such quasiconformal mappings are said to be compatible with the rational map $R$. Two quasiconformal mappings $\varphi_{1}$ and $\varphi_{2}$ are equivalent to each other if there exists a Möbius transformation $A$ such that $\varphi_{2}=A \circ \varphi_{1}$. We denote by $\operatorname{Def}(R)$ the set of equivalence classes of quasiconformal mappings $\varphi$ compatible with $R$.

The quasiconformal automorphism group $\mathrm{QC}(R)$ is the set of quasiconformal maps $\omega$ such that $R^{\omega}=R$. The group $\mathrm{QC}(R)$ acts on $\operatorname{Def}(R)$ by

$$
\omega:[\varphi] \mapsto\left[\varphi \circ \omega^{-1}\right],
$$

where $[\varphi]$ means the equivalence class of $\varphi$.

A normal subgroup $\mathrm{QC}_{0}(R)$ of $Q C(R)$ consists of quasiconformal mappings $\omega_{0} \in \mathrm{QC}(R)$ admitting a quasiconformal isotopy $\left\{\omega_{t}\right\}_{t \in[0,1]}$ in $\mathrm{QC}(R)$ such that $\omega_{1}=\mathrm{id}$.

DeFinition 4.1. The Teichmüller space of a rational map $R$ is the quotient space $\operatorname{Def}(R) / \mathrm{QC}_{0}(R)$ via the action (4.1). The quotient space $\operatorname{Mod}(R)=\mathrm{QC}(R) / \mathrm{QC}_{0}(R)$ is called the modular group (or mapping class group) and each element $[\omega] \in \operatorname{Mod}(R)$ is called a modular transformation.

Let $\omega_{1}, \omega_{2}$ be in $\mathrm{QC}(R)$. If they determines the same modular transformation, then $\omega=\omega_{1} \circ \omega_{2}^{-1} \in \mathrm{QC}_{0}(R)$. Then, we see that $\omega$ fixes each repelling periodic points of $R$. Since $\omega$ is a quasiconformal mapping of $\hat{\boldsymbol{C}}$, we conclude that $\omega$ is the identity on $J(R)$ because the set of repelling periodic points is dense in the Julia set $J(R)$ of $R$. Hence, if $\omega_{1}$ and $\omega_{2}$ determine the same modular transformation, they have the same value on the Julia set.

As for the action of the modular group, the following is known.

Proposition 4.1. The action of the modular $\operatorname{group} \operatorname{Mod}(R)$ on the Teichmüller space Teich $(R)$ is properly discontinuous.

One of the important results in $[\mathrm{MSu}]$ is a structural theorem of Teichmüller spaces of rational maps. To describe the theorem, it is necessary to understand how the Beltrami differentials of quasiconformal mappings compatible with $R$ are determined on $\hat{\boldsymbol{C}}$.

If a quasiconformal mapping $\varphi$ is compatible with a rational map $R$, then the Beltrami differential $\mu_{\varphi}$ of $\varphi$ satisfies 


$$
\mu_{\varphi}(R(z)) \overline{R^{\prime}(z)}\left(R^{\prime}(z)\right)^{-1}=\mu_{\varphi}(z)
$$

for almost all $z \in \hat{\boldsymbol{C}}$. Therefore we verify that $\mu_{\varphi}(z)$ determines $\mu_{\varphi}(w)$ for $w \in$ $\bigcup_{n=1}^{\infty} R^{-n}(z)$. It leads us the notion of the grand orbit relation with respect to the action of $R$.

Definition 4.2. The grant orbit of $z \in \hat{\boldsymbol{C}}$ is the set of $w$ such that $R^{n}(z)=$ $R^{m}(w)$ for some $n, m \geq 0$. Then, $z$ and $w$ belong to the same grand orbit equivalence class and we denote by $z \sim w$ the grand orbit equivalence relation.

From the equation (4.2), we verify that the quotient space $\hat{\boldsymbol{C}} / \sim$ is essential for Beltrami differentials of quasiconformal mappings which are compatible with $R$. Generally, the quotient space may be complicated. Fortunately, every connected component of the Fatou set $F(R)$ of $R$ is preperiodic. Namely, the following result is known.

Proposition 4.2. Every connected component $D$ of $F(R)$ is eventually periodic, that is, there exists some $n \in \boldsymbol{N}$ such that $R^{n}(D)$ is a periodic component of $R$.

This result shows that periodic components of $F(R)$ determine the Beltrami differential on $F(R)$.

A fundamental theorem of the complex dynamics gives a complete classification of periodic components of the Fatou set (cf. [CG]).

Proposition 4.3. A periodic component $D$ of the Fatou set of a rational map is one of the following types.

1. An attractive basin.

2. A super-attractive basin.

3. A parabolic basin.

4. A Siegel disk.

5. A Herman ring.

After a deep analysis on grand orbit relations on periodic components, we see that the grand orbit relations on attractive basins and parabolic basins produce Riemann surfaces of type $(1, m)$ and $(0, n)$, respectively while annuli with foliations are obtained from the grand orbit relations on super-attractive basins, Siegel disks and Herman rings. The Teichmüller spaces corresponding to the former ones are denoted by $\operatorname{Teich}\left(\Omega^{\text {dis }}, R\right)$ and the latter ones are denoted by $\operatorname{Teich}\left(\Omega^{\text {fol }}, R\right)$.

The space of Beltrami differentials supported on the Julia set $J(R)$ is identified with the set of invariant line fields. Therefore, we have the following.

THeOREM 4.4. The Teichmüller space Teich $(R)$ of a rational map $R$ is isomorphic to 


$$
M_{1}(J(R)) \times \operatorname{Teich}\left(\Omega^{\mathrm{fol}}, R\right) \times \operatorname{Teich}\left(\Omega^{\mathrm{dis}}, R\right),
$$

where $M_{1}(J(R))$ is the set of invariant line fields on the Julia set $J(R)$ of $R$. Moreover, $M_{1}(J(R))$ and Teich $\left(\Omega^{\mathrm{fol}}, R\right)$ are isomorphic to polydisks, and Teich $\left(\Omega^{\mathrm{dis}}, R\right)$ is isomorphic to finite products of Teichmüller spaces of Riemann surfaces of type $(0, n)$ and $(1, m)$.

Now, we describe a motivation of the problem treated in this section. In [Mc2], McMullen considers a holomorphic family of polynomials $P_{\lambda}$ over $\Sigma=$ $\{\lambda|10<| \lambda \mid<\infty\}\left(\cong \Delta^{*}=\{z|0<| z \mid<1\}\right)$ defined by

$$
P_{\lambda}(z)=z^{3}+\lambda z^{2} \text {. }
$$

It is shown that the family is quasiconformally stable. Hence, for any point $\lambda$ in $\Sigma$ there exists a neighbourhood $U_{\lambda}$ of $\lambda$ in $\Sigma$ such that $P_{\lambda^{\prime}}$ and $P_{\lambda}$ are quasiconformally conjugate to each other for each $\lambda^{\prime}$ in $U_{\lambda}$. Thus, we may take a quasiconformal self-mapping $f_{\lambda^{\prime}}$ of $\hat{\boldsymbol{C}}$ such that

$$
P_{\lambda} \circ f_{\lambda^{\prime}}(z)=f_{\lambda^{\prime}} \circ P_{\lambda^{\prime}}(z)
$$

for all $z \in \hat{\boldsymbol{C}}$. We take a circle $C=\left\{r e^{i \theta} \mid 0 \leq \theta \leq 2 \pi\right\} \subset \Sigma$. Then, an analytic continuation of $P_{\lambda}$ along $C$ determines a quasiconformal mapping $f_{C}$ satisfying

$$
P_{\lambda} \circ f_{C}(z)=f_{C} \circ P_{\lambda}(z) \text {. }
$$

Hence, the quasiconformal mapping $f_{C}$ determines a modular transformation $\omega_{[C]}$.

The quasiconformal mapping $f_{C}$ is not uniquely determined by $C$, but from (4.4) the restriction $\left.f_{C}\right|_{J\left(P_{\lambda}\right)}$ on the Julia set $J\left(P_{\lambda}\right)$ is uniquely determined. Moreover, $\left.f_{C}\right|_{J\left(P_{i}\right)}$ depends only on the homotopy class $[C]$ of $C$ in $\Sigma$. So, we denote it by $\omega_{[C]}^{J\left(P_{\lambda}\right)}$ and call it monodromy of the family for $[C]$ on the Julia set. Then, the following is shown ([Mc2]).

Proposition 4.5. The order of $\omega_{[C]}^{J\left(P_{\lambda}\right)}$ is infinite.

The proof is done by using an approximation of the Julia set $J\left(P_{\lambda}\right)$ via a nested sequence of closed curves in the Fatou set $F\left(P_{\lambda}\right)$ and by a careful analysis of the action of $f_{C}$ on the nested sequence. To extend Proposition 4.5, we derive a similar result by a different method to quasiconformal stable families of rational maps over the punctured disk $\Delta^{*}$.

Let $\left\{R_{\lambda}\right\}_{\lambda \in \Delta^{*}}$ be a quasiconformally stable family over the punctured disk $\Delta^{*}$. We consider a monodromy $\omega_{[C]}$ of the family for a simple closed curve $C$ around the origin. Then, we have the following theorem.

THEOREM 4.6. Let $\left\{R_{\lambda}\right\}_{\lambda \in \Delta^{*}}$ be a locally non-trivial quasiconformally stable family over the punctured disk $\Delta^{*}$. We assume that the limit $\lim _{\lambda \rightarrow 0} R_{\lambda}$ does not exist in the Teichmüller space of a rational map $R_{\lambda_{0}}$ for some $\lambda_{0} \in \Delta^{*}$. Then, the order of $\omega_{[C]}$ is infinite. 
Proof. Suppose that the order of $\omega_{[C]}$ is finite, say $k$. Take $k$-sheeted unramified covering of $\Delta^{*}$. Then, the covering surface is still the punctured disk $\Delta^{*}$. Thus, we have a quasiconformally stable holomorphic family $\left\{R_{\zeta}\right\}_{\zeta \in \Delta^{*}}$ from $\left\{R_{\lambda}\right\}_{\lambda \in \Delta^{*}}$. From the construction, the monodromy for a simple closed curve around the origin is trivial. Therefore, we see that the assignment $\Delta^{*} \ni \zeta \mapsto R_{\zeta}$ induces a holomorphic mapping $h$ of $\Delta^{*}$ to the Teichmüller space Teich $\left(R_{\zeta_{0}}\right)$ of some $R_{\zeta_{0}}$ in the family.

Here, we consider about complex analytic properties of $\operatorname{Teich}\left(R_{\zeta_{0}}\right)$. It is well known that the polydisk and the upper half plane, which are ingredients of $M_{1}\left(J\left(R_{\zeta_{0}}\right)\right) \times \operatorname{Teich}\left(\Omega^{\mathrm{fol}}\right)$, are complete with respect to the Carathéodory metrics on each spaces. Also, it is known that any finite dimensional Teichmüller space of Riemann surfaces is complete with respect to the Carathéodory metric (cf. [E], [Sh1] etc.). Therefore, Teich $\left(R_{\zeta_{0}}\right)$, which are finite products of $M_{1}\left(J\left(R_{\zeta_{0}}\right)\right) \times$ $\operatorname{Teich}\left(\Omega^{\mathrm{fol}}\right)$ and finite dimensional Teichmüller spaces of Riemann surfaces, is complete with respect to the Carathéodory metric $([\mathrm{K}])$. This implies that the origin is a removable singularity of the holomorphic mapping $h: \Delta^{*} \rightarrow$ $\operatorname{Teich}\left(R_{\zeta_{0}}\right)$ and the $\operatorname{limit} \lim _{\zeta \rightarrow 0} h(\zeta)$ exists in $\operatorname{Teich}\left(R_{\zeta_{0}}\right)$. It contradicts our assumption.

The statement of Theorem 4.6 is similar to that of Proposition 4.5, but it does not cover that of Proposition 4.5 because $\omega_{[C]}^{J\left(R_{\lambda}\right)}$ may be of finite order even if $\omega_{[C]}$ is of infinite order. Here, we extend Proposition 4.5 by the following way.

THEOREM 4.7. Let $\left\{P_{\lambda}\right\}_{\lambda \in \Delta^{*}}$ be a locally non-trivial quasiconformal stable holomorphic family of polynomials of degree $d$ over the punctured disk $\Delta^{*}$ and $C(\lambda)$ the set of finite critical points of $P_{\lambda}$. Suppose that the limit $\lim _{\lambda \rightarrow 0} P_{\lambda}$ does not exist in the Teichmüller space of a rational map $P_{\lambda_{0}}$ for some $\lambda_{0} \in \Delta^{*}$, and that the set $C(\lambda)$ has the following properties.

1. There exists a non-empty subset $A(\lambda)$ of $C(\lambda)$ such that any $c \in A(\lambda)$ is attracted to $\infty$, that is, $\lim _{n \rightarrow \infty} P_{\lambda}^{n}(c)=\infty$.

2. $C(\lambda)-A(\lambda)$ is not empty, and any $c \in C(\lambda)-A(\lambda)$ is either a superattracting periodic point or it lands in a parabolic component of $F\left(P_{\lambda}\right)$. Furthermore, if $c$ is in a parabolic component, then it is a unique critical point which lands in the component. Then, $\omega_{[C]}^{J\left(P_{\lambda}\right)}$ is of infinite order.

Remark 4.1. We have some comments about the above assumption.

- From the assumption in Theorem 4.7, we have $d \geq 3$. Furthermore, we see that the Julia set $J\left(P_{\lambda}\right)$ is not connected.

- Suppose that a holomorphic family of rational maps satisfies the above conditions (1) and (2), and that the postcritical sets of critical points in $A(\lambda)$ are disjoint to each other for any $\lambda \in \Delta^{*}$. Then the postcritical set gives a holomorphic motion on $\Delta^{*}$. It follows from Corollary 7.5 in $[\mathrm{MSu}]$ that the family is quasiconformally stable. In particular, if $A(\lambda)$ consists of only one critical point, then the family satisfying the condition (1) and (2) is quasiconformally stable. 
Proof. First of all, we note that under the condition of the theorem, every $P_{\lambda}$ is expansive in the sense of Denker and Urbański ([DU]) because the Julia set $J\left(P_{\lambda}\right)$ has no critical point ([DU] Theorem 4). Therefore, $J\left(P_{\lambda}\right)$ has zero area ([DU] Theorem 14) and the space of invariant line fields on the Julia set is trivial.

Let $f$ be a quasiconformal self-mapping of $\hat{\boldsymbol{C}}$ which determines the monodromy $\omega_{[C]}$. Since the quasiconformal self-mapping $f$ satisfies $P_{\lambda} \circ f=f \circ P_{\lambda}$, the mapping $f$ preserves both $F\left(P_{\lambda}\right)$ and the set of critical points of $P_{\lambda}$. Thus, by taking a finite-sheeted covering of $\Delta^{*}$ which is still $\Delta^{*}$ if necessary, we may assume that any component $F$ of $F\left(P_{\lambda}\right)$ with $F \cap C(\lambda) \neq \emptyset$ is preserved by $f$. So, $f$ determines modular transformations of Teichmüller spaces of Riemann surfaces and holomorphic mappings of $\boldsymbol{H}$ both of which are factors of Teich $\left(P_{\lambda}\right)$. Since $\omega_{[C]}$ is of infinite order from Theorem 4.6, at least one modular transformation on a factored Teichmüller space is of infinite order. Only Fatou components which contain forward orbits of critical points contribute to the Teichmüller space of $P_{\lambda}$. Hence, we consider only super-attractive basin at $\infty$ and Fatou components which meet with $C(\lambda)$.

Let $F$ be a component of $F\left(P_{\lambda}\right)$ with $F \cap C(\lambda) \neq \emptyset$. From our assumption, $F$ is either parabolic or super-attractive.

If $F$ is parabolic, then the set of grand orbit equivalence classes in $F$ gives the Teichmüller space of thrice punctured sphere because $F$ contains only one critical orbit. Therefore, the Teichmüller space is trivial, and we do not need to care about such a component.

If $F$ belongs to a bounded super-attractive cycle, then $F$ contains only a super-attracting periodic point in the post-critical set from our assumptions. Thus, the Teichmüller space for $F$ is also trivial and we do not need to consider it.

Hence, the rest is a super-attractive component $F_{\infty}$ of $\infty$ with critical points. As we have seen, $\omega_{[C]}$ induces a modular transformation of infinite order acting on the space of grand orbits in $F_{\infty}$. First, we assume that $\left|F_{\infty} \cap C(\lambda)\right|=1$. Then, $F_{\infty}$ contains a super-attracting fixed point $\infty$ and a critical point $c_{1}$ which is attracted to $\infty$. Consider the grand orbit equivalence classes in $F_{\infty}$. They consists of a foliated annuli bounded by leaves containing grand orbits of the critical point $c_{1}$.

To see this, take a holomorphic mapping $\varphi$ in the neighbourhood of $\infty$ with $\varphi(\infty)=0$ and

$$
\varphi\left(P_{\lambda}(z)\right)=\varphi(z)^{d} .
$$

Then, It is known that $\log |\varphi(z)|$ is defined all over $F_{\infty}$ and that it is Green's function of $F_{\infty}$ with pole at $\infty . \quad \varphi^{-1}$ has an analytic continuation along any line from 0 unless the line encounters $\varphi\left(c_{1}\right)$.

Let $\Delta_{r}=\{|z|<r\}$ be the maximal disk where $\varphi^{-1}$ is defined. In $D_{r}=$ $\varphi^{-1}\left(\Delta_{r}\right) \subset F_{\infty}, \varphi^{-1}(\{|z|=\rho\})(\rho<r)$ is a subset of the closure of the grand orbits of any $p \in \varphi^{-1}(\{|z|=\rho\})$, and $L_{1}=\partial D_{r}$ contains a union of finite number of simple closed curves $A_{1}, \ldots, A_{n}$ attached to each other at $c_{1}$. Set $L_{0}=P_{\lambda}\left(L_{1}\right)$. 


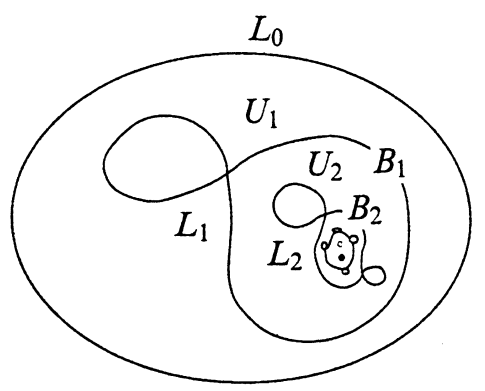

FIGURE 1

Then, $L_{0}$ is a simple closed curve and an annulus $U_{1}$ bounded by $L_{0}$ and $L_{1}$ is foliated by leaves of the closure of grand orbits equivalence classes. Actually, the leaves are level curves of $\varphi$.

From our assumption, There exist a critical point $c \notin\left\{\infty, c_{1}\right\}$ of $P_{\lambda}$ and a simple closed curve $A_{j}(1 \leq j \leq n)$ such that $c$ belongs to a component of $\hat{\boldsymbol{C}}-A_{j}$ not containing $\infty$. We denote the curve $A_{j}$ by $B_{1}$.

Notation. For any simple closed curve $C$ with $C \not \nexists \infty$, we call a component of $\hat{\boldsymbol{C}}-C$ which does not contain $\infty$ the inside of $C$ and denote it by $I(C)$.

LEMmA 4.8. There exists a component $B_{2}$ of $P_{\lambda}^{-1}\left(B_{1}\right)$ such that $I\left(B_{1}\right) \supset$ $I\left(B_{2}\right) \cup B_{2} \ni c$ and $P_{\lambda}: U_{2} \rightarrow U_{1}$ is a covering map of some degree $k \geq 2$, where $U_{2}$ is a component of $P_{\lambda}^{-1}\left(U_{1}\right)$ with $\partial U_{2} \supset B_{2}$.

Proof of Lemma 4.8. From our assumption (2), the critical point $c$ is in a forward invariant component of $F\left(P_{\lambda}\right)$. Therefore, $P_{\lambda}(c)$ is in $I\left(B_{1}\right)$. Thus, we may find a component $B_{2}$ of $P_{\lambda}^{-1}\left(B_{1}\right)$ whose inside contains $c$.

A polynomial $P_{\lambda}(z)-P_{\lambda}(c)$ has at least two zeros in $I\left(B_{1}\right)$ because $I\left(B_{1}\right)$ contains a critical point $c$. And it has no poles in $I\left(B_{1}\right)$. It follows from the argument principle that $P_{\lambda}: U_{2} \rightarrow U_{1}$ is a covering map with some degree $k>1$.

Denote by $L_{2}$ a component of $P_{\lambda}^{-1}\left(L_{1}\right)$ which contains $B_{2}$. Note that $L_{2}$ is a part of $\partial U_{2}$ and it consists of $B_{2}$ and simple closed curves, say $C_{21}, C_{22}, \ldots$, $C_{2 \ell(2)}$, which are attached to $B_{2}$ at points in $P_{\lambda}^{-1}\left(c_{1}\right)$.

We can continue the above procedure. Namely, we may find a simple closed curve $B_{n+1}$ in $I\left(B_{n}\right)$ so that $I\left(B_{n}\right) \supset I\left(B_{n+1}\right) \cup B_{n+1} \ni c$ for $n=1,2, \ldots$ Furthermore, there exists a nested sequence $\left\{U_{n}\right\}_{n=1}^{\infty}$ of annuli with $\partial U_{n} \supset B_{n}$ such that $P_{\lambda}^{n}\left(U_{n}\right)=U_{0}$ and $P_{\lambda}: U_{n+1} \rightarrow U_{n}$ is a covering map with degree $\geq 2$. We denote by $L_{n}$ a component of $P_{\lambda}^{-n+1}\left(L_{1}\right)$ which contains $B_{n}$. As before, $L_{n}$ is a part of $\partial U_{n}$ and it consists of $B_{n}$ and a finite number of simple closed curves, say $C_{n 1}, C_{n 2}, \ldots, C_{n \ell(n)}$, which are attached to $B_{n}$ at points in $P_{\lambda}^{-n}\left(c_{1}\right)$. 
Recall that $\omega_{[C]}$ induces a modular transformation of infinite order acting on the space of grand orbits in $F_{\infty}$. Since $f$ fixes the critical point $c_{1}$ in $F_{\infty}$, it also fixes any point on two simple closed curves $L_{0}$ and $P_{\lambda}\left(L_{0}\right)$. Therefore, the action on an annulus $U_{0}=P_{\lambda}\left(U_{1}\right)$ must be a Dehn twist.

On the other hand, $P_{\lambda}^{n}: U_{n} \rightarrow U_{0}$ is a covering map with degree not less than $2^{n}$ with the following commutative diagram.

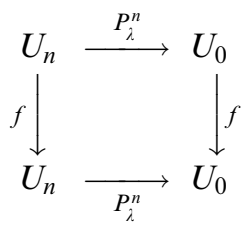

Hence, for a sufficiently large $n$ the action of $f$ on $U_{n}$ is a "fractional" Dehn twist. In other words, $f \mid U_{n}$ is regarded as a "rotation" with angle $2 \pi / N_{n}$ and $N_{n} \rightarrow \infty$ as $n \rightarrow \infty$. Therefore, it gives a permutation on $\left\{C_{n 1}, C_{n 2}, \ldots, C_{n \ell(n)}\right\}$ and the order diverges as $n \rightarrow \infty$. Since $I\left(C_{n j}\right) \cap J\left(P_{\lambda}\right) \neq \emptyset$ for each $C_{n j}$, we have shown that $\omega_{[C}^{J\left(P_{\lambda}\right)}$ is of infinite order when $\left|F_{\infty} \cap C(\lambda)\right|=1$.

When $\left|F_{\infty} \cap C(\lambda)\right| \geq 2$, we also have several foliated annuli in $F_{\infty}$ bounded by leaves containing critical points or postcritical points. Since $\omega_{[C]}$ is of infinite order, we verify that the action of $f$ is a Dehn twist on some annulus. Hence the same argument also works when $\left|F_{\infty} \cap C(\lambda)\right| \geq 2$. The details are left to the reader.

Thus, we complete the proof of Theorem 4.7.

\section{Examples}

In this section, we exhibit some examples about Theorem 4.7. The following families are defined over $\{|\lambda|>M\}$ for some $M>0$. As we noted in the remark of Theorem 4.7, the holomorphic motion formed by the postcritical set induces the quasiconformal stability of the family. Thus, we verify that all of the following families are quasiconformally stable if they satisfy the conditions (1) and (2) in Theorem 4.7.

Example 5.1. $\quad P_{\lambda}(z)=z^{d}-\lambda z^{d-1}(d \geq 3)$. have

This is a direct extension of an example given in McMullen [Mc2]. We

$$
P_{\lambda}^{\prime}(z)=d z^{d-2}\left(z-\frac{d-1}{d} \lambda\right)
$$

Thus, $z=0$ is a super-attracting fixed point and $\alpha=(d-1) \lambda / d$ is another critical point. We verify that if $M>0$ is sufficiently large, then $\alpha$ is attracted to $\infty$. Thus, the family satisfies the condition of Theorem 4.7. 
Example 5.2. For $n \geq 2$,

$$
\begin{aligned}
P_{\lambda}(z)= & \frac{1}{(n+2) \lambda-n}\left\{(n+1) n z^{n+2}\right. \\
& \left.-(n+2) n(\lambda+1) z^{n+1}+(n+2)(n+1) \lambda z^{n}\right\} .
\end{aligned}
$$

We have

$$
P_{\lambda}^{\prime}(z)=\frac{(n+2)(n+1) n}{(n+2) \lambda-n} z^{n-1}(z-1)(z-\lambda) .
$$

Hence, $z=0,1, \lambda$ are critical points and $z=0,1$ are super-attracting fixed points.

From (5.1), we have

$$
\left|P_{\lambda}(\lambda)\right|=\frac{|\lambda|^{n+1}}{|(n+2) \lambda-n|}|n \lambda-n-2| .
$$

Hence, if $|\lambda| \gg 1$, then $\left|P_{\lambda}(\lambda) / \lambda\right| \gg 1$. From

$$
\begin{aligned}
\left|P_{\lambda}(z)\right|= & \frac{|z|^{n}}{|(n+2) \lambda-n|} \\
& \times\left|n(n+1) z^{2}-n(n+1)(\lambda+1) z+(n+1)(n+2) \lambda\right| \\
\geq & \frac{|z|^{n}}{|(n+2) \lambda-n|}\left\{n(n+1)|z|^{2}-n(n+1)(|\lambda|+1)|z|\right. \\
& -(n+1)(n+2)|\lambda|\},
\end{aligned}
$$

it follows that if $|z / \lambda| \gg 1$ and $|\lambda| \gg 1$, then $\left|P_{\lambda}(z) / z\right| \gg 1$. Hence, we verify that if $M>0$ is sufficiently large, then $\lambda$ is attracted to $\infty$. Thus, the example satisfies the condition of Theorem 4.7.

Finally, we give an example with a parabolic fixed point.

Example 5.3.

$$
P_{\lambda}(z)=\frac{1}{3} z^{3}-\frac{1}{2}\left(\lambda+\lambda^{-1}\right) z^{2}+z
$$

We have

$$
P_{\lambda}^{\prime}(z)=(z-\lambda)\left(z-\lambda^{-1}\right), \quad P_{\lambda}^{\prime}(0)=1,
$$

and $P_{\lambda}(0)=0$. Hence, $z=0$ is a parabolic fixed point and $z=\lambda, \lambda^{-1}$ are critical points of $P_{\lambda}(z)$. We see that if $M>0$ is sufficiently large, then $P_{\lambda}^{n}(\lambda) \rightarrow \infty$ as $n \rightarrow \infty$. On the other hand, a parabolic component contains a critical point (cf. [CG] III. Theorem 2.3). Therefore, $z=\lambda^{-1}$ is attracted to $z=0$ and it is contained in the parabolic component for $z=0$. Thus, the example also satisfies the condition of Theorem 4.7 . 


\section{REFERENCES}

[AB] L. V. AhLFors AND L. BERs, Riemann's mapping theorem for variable metrics, Ann. of Math., 72 (1960), 385-404.

[B] A. F. Beardon, Iteration of Rational Functions, Springer-Verlag, 1991.

[CG] L. Carleson and T. W. Gamelin, Complex Dynamics, Universitext, Springer-Verlag, 1993.

[DU] M. Denker AND M. URbaŃSKI, Hausdorff and conformal measures on Julia sets with a rarionally indifferent periodic point, J. London Math. Soc. (2), 43 (1991), 107-118.

[E] C. J. EARLE, On the Carathéodory metric in Teichmüller spaces, Discontinuous Groups and Riemann Surfaces, 1973 Maryland Conference, Princeton University Press, 1974, 99-103.

[I] Y. ImayoshI, Holomorphic families of Riemann surfaces and Teichmüller spaces Riemann Surfaces and Related Topics, 1978 Stony Brook Conference, Princeton University Press, 1981, 277-300.

[ISh] Y. Imayoshi AND H. Shiga, A finiteness theorem for holomorphic families of Riemann surfaces, Holomorphic Functions and Moduli II, Springer-Verlag 1988, 207-219.

[K] S. Kobayashi, Hyperbolic Manifolds and Holomorphic Mappings, Marcel Dekker, 1970.

[MSS] R. Manẽ, P. SAD AND D. Sullivan, On the dynamics of rational maps, Ann. Sci. École Norm. Sup., 16 (1983), 193-217.

[Mc1] C. McMullen, Automorphisms of rational maps, Holomorphic Functions and Moduli I, Springer-Verlag, 1988, 31-61.

[Mc2] C. MCMullen, Families of rational maps and iterative root-finding algorithms, Ann. of Math., 125 (1987), 467-493.

[MSu] C. McMullen and D. Sullivan, Quasiconformal homeomorphisms and dynamics III: The Teichmüller space of a holomorphic dynamical system, Adv. Math., 135 (1998), 351-395.

[Sh1] H. ShigA, On analytic and geometric properties of Teichmüller spaces, J. Math. Kyoto Univ., 24 (1984), 441-452.

[Sh2] H. Shiga, On the monodromies of holomorphic families of Riemann surfaces and modular transformations, Math. Proc. Cambridge Philos. Soc., 122 (1997), 541-549.

[Su] D. Sullivan, Quasiconformal homeomorphism and dynamics I: Solution of Fatou-Julia problem on wandering domains, Ann. of Math. (2), 122 (1985), 401-418.

Department of Mathematics

Tokyo Institute of TeChNOLOGY

2-12-1 Oh-OKayama, Meguro-Ku

TOKYO, 152-8551, JAPAN

E-mail: shiga@math.titech.ac.jp 